\title{
Retrospective studies on drug utilization patterns of asthmatics in a Government hospital in Kedah, Malaysia
}

\author{
*Sam Aseer Thamby, Puah Juling, Bernice Ting Wan Xin, Ngo Chooi Jing \\ Faculty of Pharmacy, AIMST University, 08100 Bedong, Malaysia
}

\begin{abstract}
Retrospective DUE is the reviewing of drug therapy after the patient has received the medication. Asthma is a common chronic disease in Malaysia. A National Health and Morbidity Survey in 1996 indicated that asthma prevalence in Malaysia was $4.2 \%$. Statistics on the causes of death have noted asthma as one of the 10 principal causes of death $(7.3 \%)$ in Malaysia. The purpose of the study was to compare drug utilization patterns amongst the asthmatics from the past two years (since 2009) in Kedah state. Asthmatics admitted to the hospital in the preceding two years were analyzed by their patient charts. This study focused on both smoker and non smoker asthmatics. It was observed that salbutamol was most commonly prescribed, followed by montelukast and prednisolone. In the combination therapy, the most commonly prescribed was Symbicort, followed by seretide and combivent. Corticosteroids constituted the most prevalent class, followed by beta- 2 agonists, leukotriene modifiers and methylxanthines. The study presented the most recent scenario on drug utilization pattern of asthmatics in Kedah state.
\end{abstract}

Key Words: Salbutamol, Combivent, Symbicort, Turbuhaler, Nebulizer, Handihaler.

\section{INTRODUCTION}

Drug utilization evaluation / review is defined by WHO as 'marketing, distribution, prescription and use of drugs in a society, with special emphasis on the resulting medical, social and economic consequences' (Parthasarathi et al., 2004). The 3 types of DUE are Prospective; Concurrent and Retrospective. A retrospective DUE is performed after the patient has received the medication / drug therapy. It may detect patterns in prescribing, dispensing, or administering drugs to prevent recurrence of inappropriate use or abuse, and serves as a means for developing prospective standards and target intervention. Patient medical charts or computerized records are screened to determine whether the drug therapy met approved criteria, and aids prescribers in improving care for their patients, individually and group-wise.

\footnotetext{
*Corresponding Author:

Mr. Sam Aseer Thamby, Lecturer

Faculty of Pharmacy, AIMST University

08100 Bedong, Malaysia

E-mail: samthamby@gmail.com

Contact No.: +60164266240
}

Asthma is bronchial inflammation causing swelling and airways constriction, resulting in breathing difficulties. The bronchial narrowing is usually totally or at least partially reversible with treatments. Asthma is a serious global health problem. Asthma can be acute or chronic; extrinsic or intrinsic. A National Health and Morbidity Survey in 1996 indicated that the prevalence of asthma in Malaysia was $4.2 \%$ (Rugayah, 1997). The various triggers of asthma are infection, dust mites, molds and mildew, insects, pollens, pets, irritants, stress, smoke, food etc. Asthma is mainly characterized by coughing; wheezing; shortness of breath; chest tightness, pain; PEF changes; URTI. The various medications used in asthma management are:

- Inhaled corticosteroids (ICS): Fluticasone; Budesonide; Triamcinolone; Flunisolide; Beclomethasone; Mometasone

- LABAs: Salmeterol; Formoterol

- Leukotriene modifiers: Montelukast; Zafirlukast

- Mast cell stabilizers: Sodium cromoglycate, Cromolyn and Nedocromil

- Xanthines: Theophylline

- $\quad$ Quick-relief medications: SABAs; Ipratropium; Oral corticosteroids. 
Inhaler devices like pressurised standard MDIs; Aerosol inhalers; Puffers; Breath-activated MDI inhalers; Autohalers; Spacer devices; Dry powder inhalers [Accuhaler; Turbuhaler; Aerolizer]; Nebulisers are the modes for drug medication delivery (Jang, 2009).

The objectives of the study were to study the drug utilization patterns amongst asthmatic patients of all age ranges through retrospective analysis, and to collate the data obtained.

\section{LITERATURE REVIEW}

Olga Vakhno et al. (2008) conducted a study on the novel combination inhaler for mild asthma treatment. They evaluated that $33 \%$ of patients developed the intermittent mild asthma at the end of the study. The improvement in the spirometry and quality of life level values, especially in social function $(p<0.05)$ were revealed. As a conclusion, budesonide $(100 \mathrm{mg}) /$ salbutamol (200mg) combination inhaler confirmed its efficacy and safety for mild asthma maintenance treatment. It can be used in the fixed, flexible or intermittent on-demand dosage regimens (Olga, 2008).

Zuber Ahmad, MD et al. (2008) analyzed relative and additional bronchodilator response of salbutamol and ipratropium in smoker and non smoker asthmatics. Ninety six asthma patients (48 life time non-smokers and 48 current smokers), were given sequential doses of inhaled salbutamol, and after achieving maximal therapeutic effect, Ipratropium was administered to see the additional bronchodilatation. This study indicates that non-smoker asthmatics showed maximal response with salbutamol alone. Ipratropium did not give any significant additional benefit. In smoker asthmatics, neither of the two drugs was able to fully dilate airways. Both the drugs caused further dilatation when given as second drug. Ipratropium was more effective in smoker asthmatics when given as a sole agent or as a second drug (Ahmad, 2008).

Dr. Tehseen Ashraf Kohistani (2007) conducted a study on acute severe asthma by comparing the improvement in peak expiratory flow rate (PEFR) of patients using two regimens of broncho-dilator therapy which are salbutamol nebulization and salbutamol plus ipratropium bromide nebulization. Besides that, he also compared the hospital admission rates in the above mentioned two treatment groups. Sixty adult asthmatic patients with peak expiratory flow rate (PEFR) less than 200 liters per minute were randomly assigned to nebulization treatment with salbutamol or the same salbutamol regimen plus ipratropium bromide. He evaluated that the increase in PEFR over time was significantly greater in combined ipratropium plus salbutamol group $(p=0.01)$ also the proportion of admitted patients was less in combined salbutamol plus ipratropium bromide group $4 / 30$ vs $11 / 30, p=0.036$. Hence, he concluded that combined ipratropium bromide plus salbutamol nebulization was superior to salbutamol nebulization alone and it should be used in the initial management of patients who present with acute severe asthma (Kohistani, 2007).

Dr. A. Tobin (2005) reviewed the evidence for the use of intravenous salbutamol, its systemic effects and the potential complications that may occur in patients with severe asthma. He observed that intravenous salbutamol is recommended in the treatment of severe asthma when there is failure to respond to nebulised $\beta 2$-agonists. He concluded that systemic salbutamol has metabolic effects that may worsen respiratory function in asthma and should not be given by intravenous infusion to asthma patients outside of clinical trials. For patients who fail to respond to inhaled $\beta 2$-agonists, ipratropium and systemic steroids, consideration should be given to other therapies such as noninvasive ventilation rather than increasing the dose of a drug that may paradoxically worsen respiratory function (Tobin, 2005).

\section{METHODOLOGY}

This retrospective study focussed on asthmatic patients admitted in a government hospital in Kedah, Malaysia. The patients' data were collected from the available records in hospital. The study was of 3 months duration. The tentative sample size fixed was 250 patients. This sample size was obtained after employing the 'National sample size calculator for specific populations'. The data collection format was created by giving priority to the medications used by the asthmatics. The format was verified and authenticated by the government 
hospital authorities (doctors, chief pharmacists and the hospital preceptors for the students). Data was collected from randomly selected bronchial asthma patients, regardless of severity, who were treated during the preceding two years in the hospital. All the patients had an established diagnosis of bronchial asthma. The patients were treated according to the established general measurement guidelines of the hospital. The data was analyzed with respect to the medications used (dosage). The results were presented to the clinicians of the hospital.

\section{Inclusion criteria}

Asthma patients (irrespective of age, gender, smoking status and presence of concurrent disease/s) treated at the Government hospital in Kedah.

\section{Exclusion criteria}

Patients with other respiratory disorders like chronic obstructive pulmonary disease, bronchitis or emphysema and other known lung disease.

\section{RESULTS AND DISCUSSION}

\section{Monotherapy}

The most commonly prescribed drug was Salbutamol 120 (20.97\%), followed by Montelukast 89 (15.56\%) number of medication ad Prednisolone 87 (15.21\%) among the other drugs upon 572 number of medication. Salbutamol or albuterol is chosen as the first line agent in the management of asthma where they are used as required by the patient for the symptomatic relief of breathlessness and wheezing. It is the most widely prescribed bronchodilator on the control of asthma ( Reid, 2005). This is stated in the previous study done by Lee PY et al in Asthma control and prior medical care of patients presenting with acute asthma at the emergency department where it showed that salbutamol was the most prescribed drugs where fifty-seven (81\%) patients were using inhaled salbutamol but $21(30 \%)$ were still using oral short acting salbutamol. Only $32(46 \%)$ patients used inhaled corticosteroids (http://www.allergyscape.com/asthma-treatment.html).

This is because salbutamol does not increase exacerbation rates. The symptom relief attained by using salbutamol regularly may well mask inflammation. It is a short acting selective beta 2 agonist which work as a bronchodilator to improve symptoms associated with metered dose inhaler (MDI)
$113(19.76 \%)$, turbuhaler $4 \quad(0.70 \%)$, nebulizer solution $1(0.18 \%))$ and intravenous salbutamol 2 $(0.35 \%)$. However in the study, meter dose inhaler (MDI) Salbutamol $100-200 \mathrm{mcg}$ is preferred among others. As for the severe bronchospasm IV injection and nebulizer are used in emergency case, as such Salbutamol $250 \mathrm{mcg}$ (as a solution of $50 \mathrm{mcg} / \mathrm{ml}$ ) via IV injection, or via IV infusion of a solution containing $5 \mathrm{mg}$ in $500 \mathrm{ml}$ at a rate of $3-20 \mathrm{mcg} / \mathrm{min}$ is used. For those via nebulizer: $2.5-5 \mathrm{mg}$, may repeat up to 4 times daily. Alternatively, may be given continuously at a rate of 1-2 mg/hr (Dennis et al., 2000).

In adults and children with persistent asthma, Montelukast improves pulmonary function tests; decreases nocturnal awakenings and $\beta 2$-agonist use, and improves asthma symptoms (http://www.mims.com). Its ability to allow a reduction in inhaled steroids may be of relevance in patients receiving high-dose inhaled steroids. The reason that leukotriene antagonists work even in the presence of inhaled steroids is that at the doses of inhaled steroids used clinically, they cannot prevent the production of leukotriene (Wells 2011).

Prednisolone is the most effective anti-inflammatory medications for the treatment of persistent asthma (Shaughnessy 1993). Studies have demonstrated their efficacy in reducing asthma symptoms, improving quality of life, improving lung function, decreasing airway hyperresponsiveness, controlling airway inflammation, reducing frequency and severity of exacerbations and reducing asthma mortality (Bateman 2008). Oral steroids maybe required for severe chronic asthma. In the studies, Prednisolone tablet in $5 \mathrm{mg}$ and $10 \mathrm{mg}$ are used, with $5 \mathrm{mg}$ preferred over the $10 \mathrm{mg}$ as to reduce the side effects of prednisolone while obtaining it's antiinflammatory effect (Juniper 1990).

The least prescribed medications are Dexamethasone $1(0.18 \%)$ and Ipratropium bromide $1(0.18 \%)$ in the context of monotherapy. If dexamethasone is given orally or by injection (parenteral) over a period of more than a few days, side-effects common to systemic glucocorticoids may occur. These may include: Stomach upset, increased appetite leading to significant weight gain, latent diabetes mellitus often becomes manifest. Glucose intolerance is worsened in patients with preexisting 


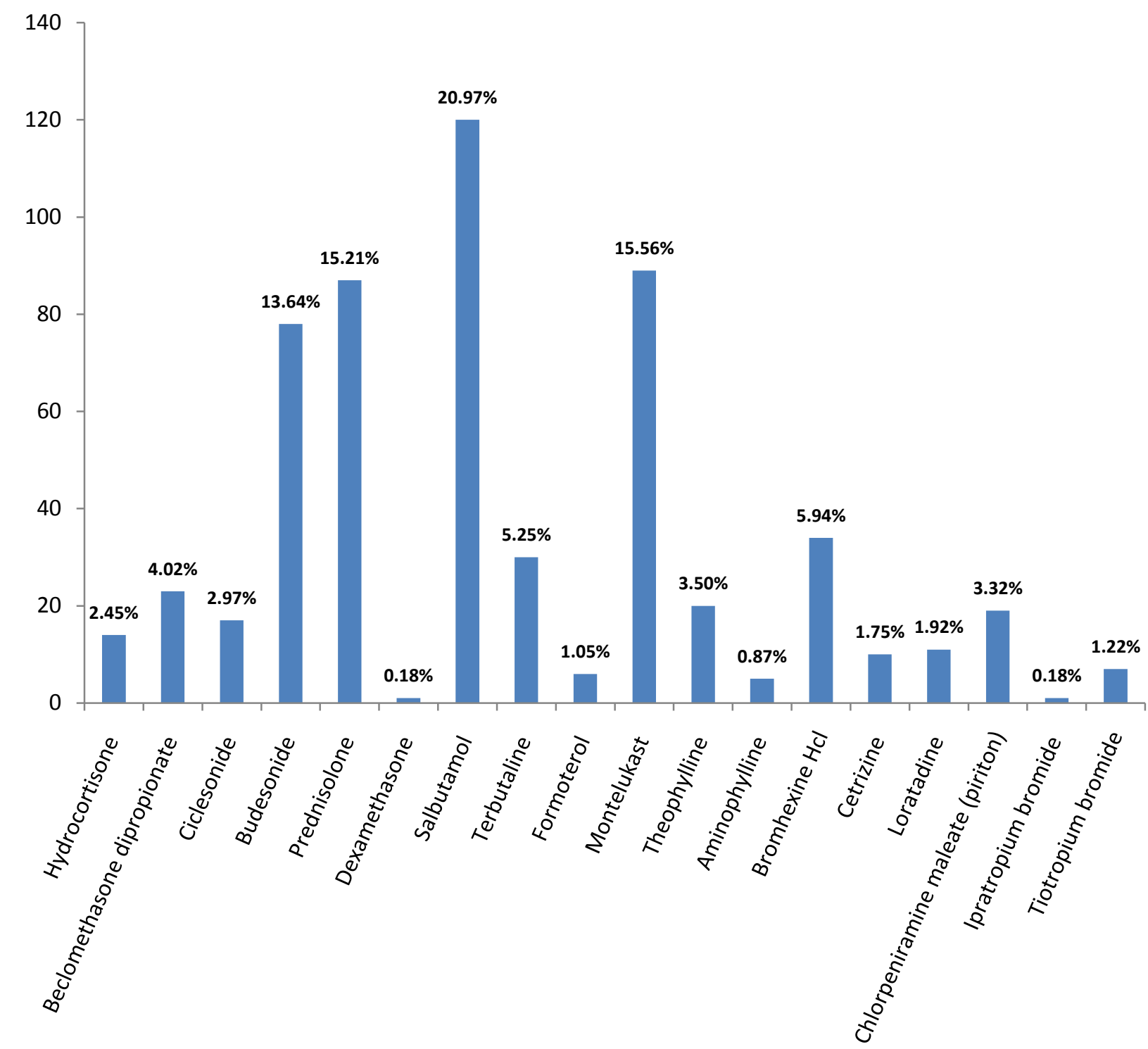

Graph I (a): Asthmatic Medications (Monotherapy).

diabetes. Osteoporosis and depression of the adrenal gland is usually seen (Pauwels 1997). However, it is contraindicating by a study which showed that Dexamethasone was more effective than hydrocortisone in the treatment of acute paediatric asthma (http://en.wikipedia.org/wiki/dexamethasone\#sideef fects). Ipratropium bromide has a major role is in the treatment of chronic obstructive pulmonary disease (Ebrahimi, 2007).

This was stated in the previous study done by John $\mathrm{K}$. Reid that Ipratropium bromide is a quaternary anticholinergic medication that is mostly useful in
COPD but even though it has a limited role in the treatment of asthma, it is a potent antagonist of methacholine, the most commonly used agent in bronchoprovocation testing for evaluation of possible asthma (Reid, 2005). It has a limited place in the day-to-day management of people with asthma, although in children the addition of ipratropium bromide to a SABA has shown benefit in the initial management of moderate and severe acute asthma. Adverse effects are primarily oropharyngeal and relate to its anticholinergic effects such as dry mouth, throat irritation (Ebrahimi, 2007). 


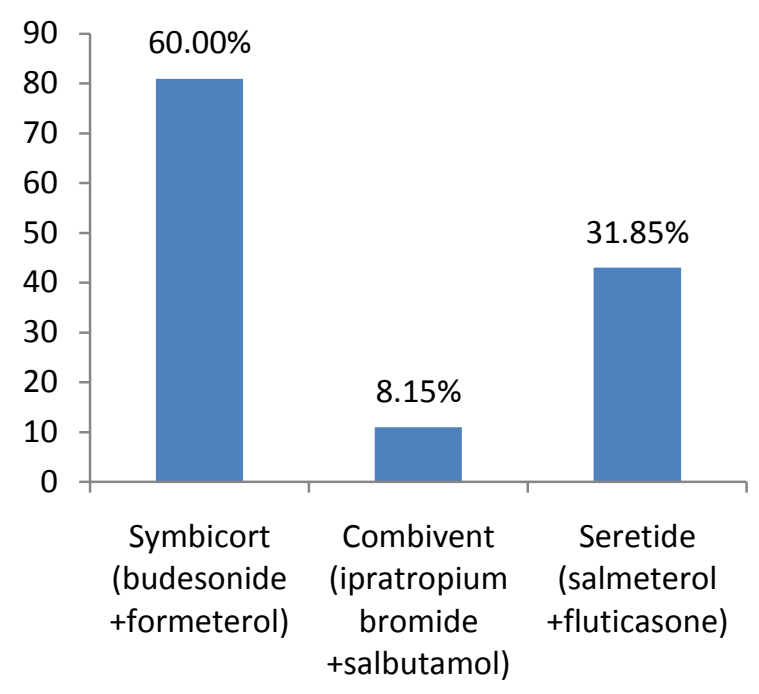

Graph I (b): Asthmatic Medications (Combination therapy).

\section{Combination therapy}

Symbicort $81^{\circledR}(60 \%)$ was the most commonly prescribed, followed by Seretide $43(31.85 \%)$ and Combivent 11 (8.15\%). The reason Symbicort constitutes the most was demonstrated in a previous study which found that SYMBICORT (budesonide/formoterol fumarate dihydrate) led to significant improvements in health-related quality of life (HRQL) and greater patient-reported satisfaction with asthma treatment, versus its monocomponents (budesonide or formoterol) or placebo (http://www.nationalasthma.org.au). It was further approved in the study done by Peters M., where he found that current control of asthma symptoms is improved or achieved with reduced total dose administration with Symbicort SMART compared with any reasonable alternate option. In every study, the risk of severe exacerbations was lower with Symbicort SMART than comparator treatment. Symbicort SMART delivers improved asthma outcomes with lower treatment and social costs than any alternative. It is a combination of budesonide and formoterol at the dose of $160 \mathrm{mcg} / 4.5 \mathrm{mcg}$ respectively which can be dispensed as MDI and turbohaler. It contains formoterol and budesonide, which have different modes of action and show additive effects in terms of reduction of asthma exacerbations. The specific properties of budesonide and formoterol allow the combination to be used both as maintenance and reliever therapy of asthma (http://www.news- medical.net/news/2007/05/24/25478). Apart from that, Symbicort is found to be cost effective among others (Wickstrøm et al., 2009). Aalber observed that Symbicort's adjustable maintenance dosing provided greater asthma control than the fixed dosing seretide (Aalber et al., 2004).

Combivent ${ }^{\circledR}$ consists of salbutamol and ipratropium bromide. Least number of cases used Combivent ${ }^{\circledR}$ $11(8.15 \%)$. This could be because Combivent inhalation aerosol is indicated for use in patients with chronic obstructive pulmonary disease (COPD) on a regular aerosol bronchodilator who continue to have evidence of bronchospasm and who require a second bronchodilator (Aalber et al., 2004). Besides, Combivent is not cost effective when compared to others.

\section{Classification}

Corticosteroids $220(38.46 \%)$ constituted the most prevalent class, followed by beta -2 agonist 156 $(27.27 \%)$, leukotriene modifiers $89(15.56 \%)$ and methyl xanthines 25 (4.37\%).

Corticosteroids constitute the most commonly used among others; this was in concordance with the study done by Tuomas Jartti who observed that Inhaled Corticosteroids are the preferred first-line controller therapy for mild to moderate persistent asthma (http://www.combivent.com). Inhaled corticosteroids were also considered the standard daily preventive treatment as it shows decrease in frequency and asthma episodes as well as the risk of asthma hospitalization (Jartti, 2008). This could be because they inhibit many components of the asthma inflammatory response. They inhibit the activation and recruitment of inflammatory cells, particularly eosinophils, $\mathrm{T}$ lymphocytes, macrophages, and dendritic cells. Although they do not block mast cell activation, they inhibit the survival of mast cells at the airway surface, thus preventing their activation in challenges such as exercise and fog. Corticosteroids also inhibit the release of mediators from structural cells in the airways, such as epithelial, smooth muscle, and endothelial cells and fibroblasts. Indeed, an inhibitory effect on epithelial cells may be one of the major actions of inhaled corticosteroids (Lee, 2001). 
Table 1: Asthmatic Medications (Dosage form).

\begin{tabular}{cll}
\hline No. & Dosage form & Number \\
\hline 1 & Metered dose inhalers (MDI) & $226(31.96 \%)$ \\
2 & Turbuhaler & $101(14.29 \%)$ \\
3 & Accuhaler & $43(6.08 \%)$ \\
4 & Nebulizer & $28(3.96 \%)$ \\
5 & Nasal spray & $5(0.71 \%)$ \\
6 & Intravenous injection & $37(5.23 \%)$ \\
7 & Tablet & $260(36.78 \%)$ \\
8 & Capsule & $7(0.99 \%)$ \\
& & Total $=\mathbf{7 0 7}(\mathbf{1 0 0 . 0 0 \% )}$
\end{tabular}

Very few anticholinergics 8 (1.4\%) were used, which could be because inhaled anticholinergics are minimally absorbed. Besides, anticholinergic bronchodilators as a class are the preferred for the treatment of COPD instead of asthma (Barnes, 1998). This was stated in an earlier study by John K. Reid et al. They observed that ipratropium bromide is a quaternary anticholinergic medication that is mostly useful in COPD but even though it has a limited role in the treatment of asthma, it is a potent antagonist of methacholine, the most commonly used agent in bronchoprovocation testing for evaluation of possible asthma (Reid, 2005).

\section{Dosage forms}

Tablets 260 (36.78\%) constituted the most common mode of administration, followed by metered dose inhaler 226 (31.96\%), turbohaler 101 (14.29\%), while the IV injections and nebulizer constitute $37(5.23 \%)$ and $28(3.96 \%)$ respectively. The least prescribed dosage form is the nasal spray $5(0.71 \%)$.

Oral tablets become necessary in patients who do not comply to the inhalation where he or she is unable to use inhaler therapy effectively especially in the group of pediatric and geriatric, mainly coordinating the beginning of inspiration with the actuation of the inhaler. This was proved in a previous study Kelloway et al who found that compliance was higher with prescribed oral medications compared with inhaled anti-inflammatory medications for asthma. It is also important to note that oral medications are not affected by inhalation technique and lung deposition that may reduce drug effectiveness. Well-designed prospective studies comparing oral and inhaled asthma thera-
Table 2: Asthmatic Medications (Classification).

\begin{tabular}{clc}
\hline No. & Category & Number \\
\hline 1 & Corticosteroids & $220(38.46 \%)$ \\
2 & Beta 2 agonists & $156(27.27 \%)$ \\
3 & Leukotriene modifiers & $89(15.56 \%)$ \\
4 & Methyl xanthines & $25(4.37 \%)$ \\
5 & Mucolytics & $34(5.94 \%)$ \\
6 & Antihistamines & $40(6.99 \%)$ \\
7 & Anticholinergics & $8(1.40 \%)$ \\
& & Total $\mathbf{5 7 2 ( 1 0 0 . 0 0 \% )}$ \\
\hline
\end{tabular}

pies could help further to determine the importance of route of administration (Gross, 2006).

The pressurized, metered dose inhaler (MDI) has the advantage of being multidose, small and widely available for most of the drugs used for asthma management. In the study, it is essential for the hospital pharmacist to dispense MDI and proper techniques of using the inhaler are taught to the patients initially, thus a large amount of MDI is being dispensed. Inhaled medicine works in the lungs right away. There are fewer side effects with inhaled medicine compared to pills or liquid forms of medicine (Kelloway, 1995). However, the effectiveness of inhaler therapy also depends on the inhaler technique. Patients may not be adequately instructed in the inhaler technique, thereby reducing the amount of drug delivered to the lungs. Cochrane, in a previous study stated 'it is important to reinforce the simple concept that failure to instruct patients on how to use inhalers and to reinforce these instructions will decrease compliance, whatever the drug or inhalation device' (Guidelines on Management of Adult Asthma a Consensus Statement of the Malaysian Thoracic Society, http://202.144.202.76/my-mts/index).

Nebulizer produces an aerosol by blowing air or oxygen through a solution to produce droplets. It requires little coordination from the patient as nay drug is inhaled through a facemask or a mouth piece using normal tidal breathing. Thus, it is useful in patients who are unable to use conventional inhaler. However, it is used most often in emergency room for an asthma attack or an uncontrollable asthma attack. The disadvantages of using a nebulizer include the long time commitment for 
maintenance treatments and lack of portability (Cochrane, 1996).

IV injections of antiasthmatic medications are also used when there is an emergency of asthma attack or uncontrollable asthma, as it gives the better absorption, and thus, fastest onset of pharmacological action of medication, apart from $100 \%$ bioavailability. It also prevents the side effect of irritation on respiratory tract (Guidelines on Management of Adult Asthma a Consensus Statement of the Malaysian Thoracic Society, http://202.144.202.76/my-mts/index).

\section{LIMITATIONS OF THE STUDY}

- Follow up could not be carried out on account of the time constraints

- Data with higher amount of authenticity can be obtained if other hospitals are also included in the study.

- Cost effectiveness of metered dose inhaler resulted in it being the most preferred dosage form.

- A significant proportion of the overall population preferred private hospitals.

\section{CONCLUSION}

The studies highlighted some interesting findings, which presents a picture of recent scenario on drug utilization pattern of asthmatic patients in Kedah. Asthma is a common chronic disease that affecting all the state in Malaysia. The statistic on the causes of death has noted asthma as one of the 10 principal causes of death in Malaysia with 7.3\% (Kohistani, 2007).

In our findings, Malays have higher prevalence rate of asthma than other ethnic groups in Malaysia. Asthmatic patients with the highest prevalence from asthma were in the age range of 50 to 59 . Geriatrics was mostly admitted for severe form of asthma, this may be due to the incorrect use of respiratory therapy kit like metered dose inhaler. There are two types of asthmatic therapy in the government hospital, which is monotherapy and combination therapy. There were a total of 572 usages of monotherapy medications. The most commonly prescribed asthmatic medication in monotherapy was Salbutamol, followed by Montelukast and Prednisolone. The most commonly prescribed combination therapy was Symbicort, followed by Seretide and Combivent.

\section{REFERENCES}

Aalbers, R. (2004). Adjustable maintenance dosing with budesonide/formoterol compared with fixed-dose salmeterol/fluticasone in moderate to severe asthma .Curr Med Res Opin, Vol. 20, p 225-240.

Ahmad, Z. (2008). To analyze relative and additional bronchodilator response of salbutamol and ipratropium in smoker and non smoker asthmatics. Chest, volume 134, pg 55003.

Barnes, P.J. (1998). Efficacy of inhaled corticosteroids in asthma. The journal of allergy and clinical immunology, volume 102 (no.4, part 1), p 531-537. PII: S00916749(98)70268-4)

Bateman, E.D (2008). Global guidelines: global strategy for asthma management and prevention: Gina Executives' summary. European Respiratory Journal, 31, p: 148-178, ERJ January 1, 2008 vol. 31 no. 1 143-178. [DOI]

Cochrane, G.M. (1996). Compliance and outcomes in patients with asthma. Drugs, 1996; 52, pg.; 12-19. [DOI]

Dennis, S.M., Sharp, S.J. \& Vickers, M.R. (2000). Regular inhaled salbutamol and asthma control: the TRUST randomised trial. Lancet, 355, pg. 1675-167. PMID:0905242

Ebrahimi, S. (2007). Comparative Efficacy of Dexamethasone versus Hydrocortisone in Severe Acute Pediatric Asthma. Iran J Allergy Asthma immunol, 6(3), pg.159-160. [DOI]

Gross, N.J. (2006). Anticholinergic agents in asthma and COPD. European Journal of Pharmacology, Volume 533, Issues 1-3, Pg. 36-39. [DOI]

Guidelines on management of adult asthma a consensus statement of the Malaysian thoracic society. [Link]

http://asthma.symbicort.com/_mshost7311943/content/840160 8/Turbuhaler16 02

http://en.wikipedia.org/wiki/Dexamethasone\#Side_effects.

http://www.allergyescape.com/asthma-treatment.html

http://www.combivent.com/

http://www.nationalasthma.org.au/cms/content/view/195/1/

http://www.news-medical.net/news/2007/05/24/25478.aspx

Jang, A.S. (2009). The impact of smoking on Clinical and Therapeutic Effects in Asthmatics: J Korean Med Sci., volume 24, issue 2, p.209-214. [DOI]

Jartti, T. (2008). Inhaled corticosteroids or montelukast as the preferred primary long-term treatment for pediatric 
asthma? European journal of pediatrics, volume 167 (7). PMID: 18214538

Juniper, E.F. (1990). Effect of long-term treatment with an inhaled corticosteroid (budesonide) on airway hyperresponsiveness and clinical asthma in nonsteroid dependent asthmatics. Am Rev Respir Dis, 142, pg. 832-836. PMID: 2221590.

Kelloway, J.S. (1995). Comparison of patients' compliance with prescribed oral and inhaled asthma medications. Arch Intern Med., volume 155(5): p 547-8. PMID: 8002686

Kohistani, T.A. (2007). Acute severe asthma: Acute Severe Asthma; Salbutamol plus Ipratropium Bromide Nebulization Versus Salbutamol Nebulization Alone. Professional Med J, volume 14, issue4: p.586-590. (Prof- 1153); Issn: 10248919.

Lee, J.H. (2001). Evaluating Asthma Medication Use Before and After an Acute Asthma-related Event. J Managed Care Pharm, p 303-08.

MIMS (Monthly Index of Medical Specialties) of Malaysia. http://www.mims.com

Olga, V. (2008). Novel combination inhaler for mild asthma treatment. Room New York, 2, Volume 14:45,489.

Parthasarathi, G., Nyfort-Hansen, K., \& Nahata. M.C. (2004). A Textbook of Clinical Pharmacy Practice1st edition; p.363. Orient Longman Private Limited.
Pauwels, R.A. (1997). Effect of inhaled formoterol and budesonide on exacerbations of asthma. Formoterol and Corticosteroids Establishing Therapy (FACET) International Study Group. N Engl J Med, 337, p.1405-1411. PMID:9358137

Reid, J.K. (2005). The Effect of Ipratropium Nasal Spray on Bronchial Methacholine Challenge. CHEST, vol.128 no.3, pg.1245-1247. [DOI]

Rugayah, B. (1997). Report of the Second National Health and Morbidity Survey Conference, Ministry of Health Malaysia. Asthma, vol.11, pg. $94-98$.

Shaughnessy, O. (1993). Differential effects of fluticasone propionate on allergen-evoked bronchoconstriction and increased urinary leukotriene E4 excretion. Am. Rev.Respir. Dis., 147, p.1472-1476. PMID: 8389108

Tobin, A. (2005). Intravenous Salbutamol: Too Much of a Good Thing? Critical Care and Resuscitation, volume 7, pg.119-127.

Wells, B (2011). Pharmacotherapy handbook 8th edition. Section 15: respiratory disorder- chapter 80 asthma. Pg.: 906-920. (ISBN13: 9780071748346).

Wickstrøm, J. (2009). Cost-effectiveness of budesonide/formoterol for maintenance and reliever asthma therapy in Denmark--cost-effectiveness analysis based on five randomised controlled trials. Clin Respir J, 3(3), p 16980. PMID:20298400 